\title{
Voice challenge in transgender women: trans women self-perception of voice handicap as compared to gender perception of naïve listeners
}

\author{
Jeanne Gabriele Schmidt(t) \\ Bárbara Niegia Garcia de Goulart ${ }^{(1)}$ \\ Maria Elza Kazumi Yamaguti Dorfman(2) \\ Gabriel Kuhl(2) \\ Lauren Medeiros Paniagua (2)
}

(1) Universidade Federal do Rio Grande do Sul, Porto Alegre, Rio Grande do Sul, Brasil.

(2) Hospital de Clínicas de Porto Alegre, Porto Alegre, Rio Grande do Sul, Brasil. Research developed at Universidade Federal do Rio Grande do Sul - UFRGS Porto Alegre, Rio Grande do Sul, Brasil, in a partnership with Hospital de Clínicas de Porto Alegre - HCPA, Porto Alegre, Rio Grande do Sul, Brasil.

Conflict of interest: Nonexistent

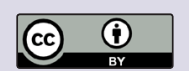

Received on: February 6, 2017 Accepted on: October 23, 2017

Mailing address:

Bárbara Niegia Garcia de Goulart Universidade Federal do Rio Grande do Sul Rua Ramiro Barcelos, 2777, sala 307 CEP 90035-003 - Porto Alegre, Rio Grande do Sul, Brasil

E-mail: bngoulart@gmail.com

\section{ABSTRACT}

Purpose: to analyze the self-perception of transgender women's voice handicap in comparison to the voices' gender perception by naïve listeners.

Methods: 31 transgender women, who were first submitted to vocal acoustic assessment and had their voices recorded to measure fundamental frequency and standard deviations, were eligible to the study. Next, they answered to the Voice Handicap Index protocol, from which, at the end of data collection, the most suited questions to the daily demands of this population were selected. Subsequently, 50 naïve blindfolded listeners were exposed to the recordings and had to identify the voices as males, females or undefined. Descriptive statistics were applied to speakers' characteristics and the Spearman's correlation coefficient was applied to the protocol scores and the speaker's voice identifications.

Results: the mean fundamental frequency found was $172.40 \mathrm{~Hz}(\mathrm{SD}=4.8 \mathrm{~Hz})$ and one third of the transgender women reported being satisfied with their voices. A moderate positive significant correlation was found in voices considered as males, and strongly significant and negative in voices considered as females. For the voices considered undefined, however, no significant correlation was found.

Conclusion: transgender women's self-perception of voice handicap is directly related to naïve listeners' perception of their voice gender.

Keywords: Voice; Transgender Persons; Gender Identity; Self Concept; Social Perception 


\section{INTRODUCTION}

Transgender people do not identify themselves as belonging to their biological gender. Among all the issues faced by this population, voice gender identification could be considered the main one, especially by transgender women.

Voice is an important gender indicator, although it is a secondary sexual feature ${ }^{1,2}$ and is relevant to transgender women, since it can be a gender "betrayer" or the last challenge to their gender adequacy towards the society ${ }^{3}$. A male-to-female gender transition requires more complex voice adjustments in order to suit the voice to the new social identity. A male voice has resonance standards, speech speed, loudness, pitch and suprasegmental features that differ from the female's voice ${ }^{4,5}$.

The manner how somebody uses the communication is influenced by other people perception and how these other people interact with them ${ }^{6}$, as well as by secondary factors such as prosody, articulatory peculiarities and vocabulary ${ }^{7}$. These characteristics also influence on voice perception ${ }^{7}$. To define the speaker's' gender multiple aspects, like culture and accent, are taken into account, since there is an association between voice perception and the person's speech and language perception ${ }^{8}$. Even if a voice does not sound familiar, the listener may have impressions about the gender, height, weight and other aspects of the speaker ${ }^{9}$.

Nowadays, transgender people have been looking for more adequate vocal quality, specially the trans women. They represent the biggest part of this population (in a ratio of three to one) ${ }^{10}$, even known that the prevalence of transgender people among literature is uncertain due to different methods applied to the researches, sociodemographic and cultural diversity 11. Even when they undergo long term hormonal treatments, changes in the voice are null, because hormones cannot heighten the fundamental frequency of voice, reduce vocal fold mass or take to long-lasting effects on pitch ${ }^{7,12,13}$. Therefore, transgender women usually seek to adjust their voices through speech therapy and surgeries, such thyroplasty, aiming a vocal quality enough for being identified as female on daily situations, especially those that does not give any visual clue to the listener, for example, during a phone call ${ }^{11}$.

Transgender people have their voices daily judged by people with no experience in voice analysis and such judgment would have a significant impact on the transgender people integration into their new gender identity ${ }^{14}$. A study ${ }^{14}$ investigated the relationship between the fundamental frequency, feeling of femininity and satisfaction of transgender people with their own voice and concluded that their happiness was not directly related to voice fundamental frequency or perception of femininity in their voice by voice professionals and naïve listeners. However, a moderate to strong correlation was found between their own perception of femininity and that of naïve listeners.

On the other hand, another study ${ }^{3}$ stated that the relationship between the transgender woman's voice gender categorization by other listeners and by herself, as well the impact on their physical, social, and emotional welfare, remains unclear. The authors emphasized that self-perception about quality of voice is essential to a successful transition ${ }^{3}$. Having their voices perceived as female would also increase selfperception about quality of life ${ }^{3}$.

The mentioned studies above ${ }^{3,14}$ were conducted in developed countries and demonstrated that an adequate voice to gender identity implies on better quality of life. There are no known studies with populations in developing countries, where the treatment is completely offered by the public health system and there is a lack of available and validated instruments to measure the general population satisfaction about their voice quality of life or voice handicap.

Therefore, the trans women voice must be analyzed according to their own perspective and the perspective of naive listeners, especially those who do not have any prior knowledge about the transgender origin of the listened voice, in order to achieve successful gender identification in society. Thus, the current study analyzes the relationship between the Brazilian transgender women voices and their identification with the female gender by comparing their own handicap perception of their voices to naïve listeners' blind perception of the individual's' gender identity and speech context.

\section{METHODS}

All the transgender women selected to the study were informed about the purpose of the research and signed the Informed Consent Form in the beginning of the data collection (brief clinical interview with each transgender women and voice gender assessment of the transgender women). The Research Ethics Committee of Hospital de Clínicas de Porto Alegre approved the present research, under the number 03-090. 
This is a descriptive cross-sectional study, in which transgender patients participating on the Gender Identity Disorder Program (PROTIG) from an university hospital in southern Brazil were selected through convenience sample. The inclusion criteria was being a transgender women assisted by PROTIG and had agreed to participate of the research during the data collection period, independently on age or have undergone surgeries or speech therapy. Data collection ran between the years 2005 and 2012 .

Initially, a brief clinical examination was performed on each transgender women, consisting on reporting impressions about their own voices (through answering simple questions as "Do you like your voice?" and "What do you think about your voice?") and answering questions about having previous laryngoscopy exam, thyroplasty surgery and voice therapy. After, their voices were recorded (sustained emission of $/ \varepsilon /$ vowel and counting from 1 to 20) using a MiniDisc Sony MZ-R50.
The recordings were subjected to vocal acoustic analysis to be featured according to the fundamental frequency (mean and standard deviation) utilizing the software Vocalgram 1.0 by CTS Informática.

Finally, to verify their general satisfaction and possible handicap assigned to their voice daily using and functions, they answered to the Voice Handicap Index (VHI) ${ }^{15,16}$. Although $\mathrm{VHI}$ is usually applied to verify voice handicap, it is also a proper instrument to measure psychosocial aspects of voice disorders. Therefore, some of the $\mathrm{VHI}$ questions were considered, according to self-perception and the perception from specialists involved in the study, based on a pilot study, more related to the voice demands of the transgender women, and then selected to be analyzed (Figure 1). These questions are more specific about how the individual's perception about their voices relates to the gender identity.

\section{Functional}

6: I use the phone less often than I would like.

8: I tend to avoid groups of people because of my voice.

11: I speak with friends, neighbors, or relatives less often because of my voice.

16: My voice difficulties restrict my personal and social life.

19: I feel left out of conversations because of my voice.

22: My voice problem causes me to lose income.

\section{Physical}

14: I feel as though I have to strain to produce voice.

17: The clarity of my voice is unpredictable.

18: I try to change my voice to sound different.

20: I use a great deal of effort to speak.

26: My voice "gives out" on me in the middle of speaking.

\section{Emotional}

7: I'm tense when talking with others because of my voice.

23: My voice problem upsets me.

24: I am less outgoing because of my voice problem

25: My voice makes me feel handicapped.

27: I feel annoyed when people ask me to repeat

28: I feel embarrassed when people ask me to repeat.

29: My voice makes me feel incompetent.

30: I'm ashamed of my voice problem.

Figure 1. Voice Handicap Index (VHI) selected questions 
The recorded voices were presented to 50 individuals, undergraduate Pharmacy students, in a silent room. They were asked to identify, in a proper multiple choice auditory-perceptive analysis instrument developed to the study, the speaker's gender as male, female or undetermined (when the voice lead to gender confusion). Listeners were not informed about the purpose of the research or exposed to visual tips in order to avoid answering induction. From their answers could be obtained the percentage of voices considered undefined, female and male to each speaker.

Descriptive statistics were performed to characterize transgender voice features, their self-perception of their own voices and the naive listeners' perception about transgenders' voices by calculating the mean and 95\% confidence interval, median and interquartile range.
The Shapiro-Wilk normality test and the Spearman coefficient ${ }^{17,18}$ were applied to estimate the concordance percentage between self-perception and naïve listeners' perception about transgender voices to a significance level of $1 \%(p \leq 0.01)$. Data were analyzed via SPSS v. 17.

\section{RESULTS}

Thirty-one trans women with ages ranging from 17 to 59 years old were considered eligible for the study. From them, 12 (38.7\%) were between 30 and 39 years, nine $(29.0 \%)$ underwent laryngoscopy exam, three $(9.7 \%)$ underwent thyroplasty surgery and only two $(6.5 \%)$, voice therapy (Table 1). The mean fundamental frequency found was $172.4 \mathrm{~Hz}(\mathrm{SD}=4.8 \mathrm{~Hz} ; 95 \% \mathrm{Cl}$ 170.9-174.1).

Table 1. Clinical characteristics of the studied transgender woman

\begin{tabular}{ccc}
\hline Age (years) & $\mathbf{n}$ & \% \\
$17-20$ & & 12.9 \\
$21-29$ & 4 & 35.5 \\
$30-39$ & 11 & 38.7 \\
40 or more & 12 & 6.5 \\
No answer & 2 & 6.5 \\
\hline Laryngoscopy & 2 & \\
Yes & 9 & 29.0 \\
No & 22 & 71.0 \\
\hline Thyroplasty & 3 & 9.7 \\
Yes & 28 & 90.3 \\
No & 2 & 6.5 \\
\hline Voice therapy & 29 & 93.5 \\
Yes & & \\
No & & \\
\hline
\end{tabular}

Among the total of the participants, nine reported to like their own voices and three, not to like it. One participant affirmed to partially like her voice; one said that her self-perception changes. Two participants reported to have had their voices identified as that of an "old lady". None of them reported previous voice problems.

The mean $\mathrm{VHI}$ score found was $18.9(\mathrm{SD}=17.4$; $95 \% \mathrm{Cl}$ 12.8-25.0; minimum $=0$; maximum $=56$ ). To the Functional questions, the mean score was $4.9(\mathrm{SD}=4.9$; minimum $=0$; maximum $=17$ ), to the Physical, was $6.4(\mathrm{SD}=5.2$; minimum $=0$; maximum $=18)$ and to the Emotional, 6.9 ( $\mathrm{SD}=7.7$; minimum $=0$; maximum $=23$ ). About the frequencies of the answers to the VHI questions, none of the trans women answered "always" to the questions, explaining the reason this answer does not appear on Table 2. The question with more answer "Almost always" was the $30^{\text {th }}$ ("I'm ashamed of my voice problem"). 
Table 2. Frequencies of the answers to the Voice Handicap Index (VHI) questions

\begin{tabular}{|c|c|c|c|c|c|c|c|c|}
\hline \multirow{2}{*}{ Questions } & \multicolumn{2}{|c|}{ Never (0) } & \multicolumn{2}{|c|}{ Almost never (1) } & \multicolumn{2}{|c|}{ Sometimes (2) } & \multicolumn{2}{|c|}{ Almost always (3) } \\
\hline & $n$ & $\%$ & $n$ & $\%$ & $n$ & $\%$ & $n$ & $\%$ \\
\hline \multicolumn{9}{|l|}{ Functional * } \\
\hline 1 & 25 & 80.6 & 5 & 16.1 & 0 & 0.0 & 1 & 3.2 \\
\hline 3 & 16 & 51.6 & 7 & 22.6 & 6 & 19.4 & 2 & 6.5 \\
\hline 5 & 28 & 90.3 & 3 & 9.7 & 0 & 0.0 & 0 & 0.0 \\
\hline 6 & 20 & 64.5 & 5 & 16.1 & 1 & 3.2 & 5 & 16.1 \\
\hline 8 & 19 & 61.3 & 7 & 22.6 & 1 & 3.2 & 4 & 12.9 \\
\hline 11 & 23 & 74.2 & 7 & 22.6 & 1 & 3.2 & 0 & 0.0 \\
\hline 12 & 16 & 51.6 & 9 & 29.0 & 4 & 12.9 & 2 & 6.5 \\
\hline 16 & 18 & 58.1 & 5 & 16.1 & 2 & 6.5 & 6 & 19.4 \\
\hline 19 & 27 & 87.1 & 0 & 0.0 & 2 & 6.5 & 2 & 6.5 \\
\hline 22 & 30 & 96.8 & 0 & 0.0 & 0 & 0.0 & 1 & 3.2 \\
\hline \multicolumn{9}{|l|}{ Physical * } \\
\hline 2 & 20 & 64.5 & 10 & 32.3 & 1 & 3.2 & 0 & 0.0 \\
\hline 4 & 9 & 29.0 & 16 & 51.6 & 3 & 9.7 & 3 & 9.7 \\
\hline 10 & 22 & 71.0 & 6 & 19.4 & 2 & 6.5 & 1 & 3.2 \\
\hline 13 & 7 & 22.6 & 21 & 67.7 & 3 & 9.7 & 0 & 0.0 \\
\hline 14 & 20 & 64.5 & 9 & 29.0 & 1 & 3.2 & 1 & 3.2 \\
\hline 17 & 13 & 41.9 & 12 & 38.7 & 2 & 6.5 & 4 & 12.9 \\
\hline 18 & 14 & 45.2 & 11 & 35.5 & 2 & 6.5 & 4 & 12.9 \\
\hline 20 & 25 & 80.6 & 3 & 9.7 & 1 & 3.2 & 2 & 6.5 \\
\hline 21 & 22 & 71.0 & 6 & 19.4 & 1 & 3.2 & 2 & 6.5 \\
\hline 26 & 22 & 71.0 & 5 & 16.1 & 2 & 6.5 & 2 & 6.5 \\
\hline \multicolumn{9}{|l|}{ Emotional * } \\
\hline 7 & 11 & 35.5 & 11 & 35.5 & 6 & 19.4 & 3 & 9.7 \\
\hline 9 & 26 & 83.9 & 4 & 12.9 & 1 & 3.2 & 0 & 0.0 \\
\hline 15 & 22 & 71.0 & 5 & 16.1 & 3 & 9.7 & 1 & 3.2 \\
\hline 23 & 15 & 48.4 & 8 & 25.8 & 1 & 3.2 & 7 & 22.6 \\
\hline 24 & 16 & 51.6 & 8 & 25.8 & 2 & 6.5 & 5 & 16.1 \\
\hline 25 & 17 & 54.8 & 10 & 32.3 & 0 & 0.0 & 4 & 12.9 \\
\hline 27 & 19 & 61.3 & 5 & 16.1 & 1 & 3.2 & 6 & 19.4 \\
\hline 28 & 19 & 61.3 & 6 & 19.4 & 1 & 3.2 & 5 & 16.1 \\
\hline 29 & 26 & 83.9 & 3 & 9.7 & 1 & 3.2 & 1 & 3.2 \\
\hline 30 & 17 & 54.8 & 6 & 19.4 & 0 & 0.0 & 8 & 25.8 \\
\hline
\end{tabular}

**None of the transgender women chose the option "Always" (4).

*Functional questions:

1: People have trouble hearing me because of my voice.

3: People have difficulty understanding me in noisy places.

5: My family has a hard time hearing me when I call for them from another room in the house.

6: I use the phone less often than I would like.

8: I tend to avoid groups of people because of my voice.

11: I speak with friends, neighbors, or relatives less often because of my voice.

12: People ask me to repeat what I say when we talk personally.

16: My voice difficulties restrict my personal and social life.

19: I feel left out of conversations because of my voice.

22: My voice problem causes me to lose income.

\section{*Physical questions:}

2: I run out of air when I speak.

4: My voice varies during the day.

10: People ask: "What do you have in your voice?".

13: My voice seems hoarse and dry.
14: I feel as though I have to strain to produce voice.

17: The clarity of my voice is unpredictable.

18: I try to change my voice to sound different.

20: I use a great deal of effort to speak.

21: My voice is worse at the end of the day.

26: My voice "gives out" on me in the middle of speaking.

\section{*Emotional questions:}

7: I'm tense when talking with others because of my voice.

9: People seem irritated with my voice.

15: I think other people do not understand my voice problem.

23: My voice problem upsets me.

24: I am less outgoing because of my voice problem.

25: My voice makes me feel handicapped.

27: I feel annoyed when people ask me to repeat.

28: I feel embarrassed when people ask me to repeat.

29: My voice makes me feel incompetent.

30: I'm ashamed of my voice problem. 
The vocal gender analysis done by non-specialists listeners (68\% female) identified 20 voices (64.5\%) as female, eight $(25.8 \%)$ as male, and three $(9.7 \%)$, as undefined (it was not possible to identify the speaker's' gender).

Data related to voice handicap perceived by the transgender women and to the gender classification of their voices by naïve listeners were correlated (Table 3). There was not significative correlation with the $\mathrm{VHI}$ to voices considered as undefined $\left(r_{s}=0.320\right.$; $p=0.079$ ), once the higher is the percentage of those voices, the higher is the IDV score. Moderate positive significant correlations were detected among voices identified as male in the VHI $\left(r_{s}=0.584 ; p=0.001\right)$ in comparison to the VHI scores related to the auditoryperceptive analysis, demonstrating that higher $\mathrm{VHI}$ scores are significantly correlated to higher auditoryperceptive percentages to voices considered as male. These findings reflect stronger vocal handicap, as well as feelings of voice inadequacy related to gender within individuals mostly identified as male. As for the $\mathrm{VHI}$ results referring to voices identified as female, the significant correlations were strong and negative $\left(r_{s}=\right.$ -0.605; $p<0.001$ ), demonstrating that low $\mathrm{VHI}$ scores are significantly correlated to lower voice handicap as well as more positive feelings about the correlation between gender and voice within individuals mostly identified as female.

Table 3. Analysis of the VHI comparing to the auditory-perceptive analysis of voices

\begin{tabular}{ccc}
\hline \multirow{2}{*}{ Perceptive-auditory analysis } & & VHI Total \\
\cline { 2 - 3 } & $\mathbf{r}_{\mathbf{s}}$ & P value \\
\hline Undefined & 0.320 & 0.079 \\
Male & 0.584 & 0.001 \\
Female & -0.605 & $<0.001$ \\
\hline
\end{tabular}

*Spearman's correlation;

**Significance level $1 \%(p \leq 0.01)$.

\section{DISCUSSION}

The current study analyzed the relationship between the self-perception of transgender women about their own voices and the gender perception of their voices by naïve listeners. The main results expound that those considering their voices adapted to the desired gender, reporting satisfaction about their own voices and, consequently, having low scores on $\mathrm{VHI}$ had their voices most identified as females by the naïve listeners. On the other hand, those participants with complaints about their voice quality and adequacy to the gender identity, presenting bigger $\mathrm{VHI}$ scores and bigger handicap, had their voices most identified as from male speakers.

None of the transgender participants referred voice problems during the initial clinical examination, which could suggest the reason for the low rates of participants that previously underwent laryngoscopy exam, thyroplasty and voice therapy. Only ten individuals were submitted to the laringoscopy, even though is strictly recommended before starting voice therapy ${ }^{3}$.

Another interesting finding was the mean fundamental frequency among the studied individuals, at
$172.4 \mathrm{~Hz}$, below the minimal frequency considered necessary by literature to identify a voice as female ${ }^{19,20}$. It is known that to consider a voice as female the frequency alone is not enough, because there are gender-specific inflections that do not change with fundamental frequency increasing 7 , which could explain the fact that one third of the participants had their voices identified as female speakers. Also, it demonstrates that the fundamental frequency is not determinant to a transgender woman consider her voice adequate to the gender, agreeing with another study ${ }^{11}$.

The VHI scores were mostly low, even when comparing the entire test with the selected questions 13.4 in the selected questions $(S D=14.3$; minimum $=0$; maximum $=42$ ), demonstrating the general satisfaction of the studied population with their own voices. The little difference between the entire test and the selected questions were due to physical-related questions concerning the use of the voice, such as fatigue, not investigated in the current study. Also, it presumes that the differences between those scores could result on changes in the final handicap score. 
Likewise, no individual answered the option "always" to the questions. The Emotional category of the $\mathrm{VHI}$ presented the highest scores, demonstrating that emotional issues affect the voice perception to gender adequacy more than the physical or the functional issues.

For the voices considered undefined for gender was not possible to establish a significant correlation when considering the entire test. From these findings is possible to infer that the transgender women have certain handicap self-perception related to their activities of voice use. It demonstrates into a listener's perception that something is "different". In these cases the voice does not properly identified the gender of the speaker.

The understanding of the daily situations faced by transgender woman using their voices is a major help to understand their needs ${ }^{11}$. The $\mathrm{VHI}$ encompass frequent issues and voice situations on the daily life of the transgender people, is validated and widely used in the country, as well by the gender transition monitoring service were the studied population were selected, what justify its applying to this study. Thus, this instrument could be easily compared to the TSEQ (considered the ideal instrument to assess the transgender voice) ${ }^{21}$ due to both use the same measure scale ${ }^{22}$. Despite the previously described on literature ${ }^{23}$, the current study demonstrated $\mathrm{VHI}$ as a valid tool to assess the transgender woman's voice.

There are some limitations in the present study. The data collection was performed in one of the few gender transition monitoring services spread across the country, what could explain the reason most participants referred to be satisfied about their voices, as well the great amount of VHI low scores. In a trans women population without specialized monitoring, the results could be different, because they could not receive the proper support to psychiatric and physiological issues involving transgenderity. This is a pioneer study in Brazil using the $\mathrm{VHI}$ to assess transgender voice handicap. There are few studies encompassing transgender voice handicap data in developing countries, where the resources for health are limited. In Brazil, the transgender transition treatment is offered by the public health system.

Voice adequacy is one of the mainly issues transgender women face during the transition, their satisfaction or dissatisfaction with it comes to be an important parameter on how the politics of gender transition are working in the country. Furthermore, the naïve listeners' assessment of the transgender voices comes to be another parameter, simple and cheap, of gender adequacy assessment to this population, as well as the quality and the efficiency of the transition treatment offered by the Brazilian public health system.

\section{CONCLUSION}

The current study shows that transgender women's self-perception about voice and gender identity are directly related to the perception of naïve listeners about these individuals' voices being male and female, especially when the listeners do not have access to other tips that may help identify the speaker's gender. As for the voices considered to be undefined, further studies with bigger samples are necessary.

\section{ACKNOWLEDGEMENTS}

To Heather Beckius for the English revision.

\section{REFERENCES}

1. Mastronikolis NS, Remacle M, Biagini M, Kiagiadaki D, Lawson G. Wendler glottoplasty: an effective raising surgery in male-to-female transexuals. $J$ Voice. 2013;27(4):516-22.

2. Sandmann K, Zehnhoff-Dinnesen A, Schmidt $\mathrm{CM}$, Rosslau K, Lang-Roth R, Burgmer $\mathrm{M}$ et al. Differences between self-assessment and external rating of voice with regard to sex characteristics, age, and attractiveness. J Voice. 2014;28(1):128. e11-28.e18.

3. Hancock A, Krissinger J, Owen K. Voice perceptions and quality of life of transgender people. J Voice. 2011;25(5):553-8.

4. Carew L, Dacakis G, Oates J. The effectiveness of oral resonance therapy on the perception of femininity of voice in male-to-female transsexuals. J Voice. 2007;21(5):591-603.

5. Gelfer MP, Tice RM. Perceptual and acoustic outcomes of voice therapy for male-to-female transgender individuals immediately after therapy and 15 months later. J Voice. 2013;27(3):335-47.

6. Hancock A, Garabedian LM. Transgender voice and communication treatment: a retrospective chart review of 25 cases. Int Journal of Lang Comm Disord. 2013;48(1):54-65.

7. Neumann K, Welzel C. The importance of the voice in male-to-femaletranssexualism. $\mathrm{J}$ Voice. 2004;18(1):153-67. 
8. Miller CL. Developmental changes in male/female voice classification by infants. Infant Behav Dev. 1983;6(3):313-30.

9. Mullenix JW, Johnson KA, Topcu-Durgun M, Farnsworth LM. The perceptual representation of voice gender. J Acoust Soc Am. 1995;98(6):3080-95.

10. Van Kesteren PJ, Gooren LJ, Megens JA. An epidemiological and demographic study of transsexuals in the Netherlands. Archives of Sexual Behavior. 1996;25(6):589-600.

11. Schwarz K, Fontanari AMV, Mueller A, Costa AB, Soll $B$, Silva DC et al. Transsexual Voice Questionnaire for Male-to-female Brazilian Transsexual People. J Voice. 2017;31(1):120.e15-120.e20.

12. Spiegel JH. Phonosurgery for pitch alteration: feminization and masculinization of the voice. Otolaryngol Clin North Am. 2006;39(1):77-86.

13. Hancock A, Colton L, Douglas F. Intonation and gender perception: applications for transgender speakers. J Voice. 2014;28(2):203-9.

14. McNeill EJM, Wilson JA, Clark S, Deakin J. Perception of voice in the transgender client. J Voice. 2008;22(6):727-33.

15. Jacobson HB, Johnson A, Grywalski C, Silbergleit $A K$, Jacobson GP, Benninger $M$ et al. The Voice Handicap Index (VHI): development and validation. Amer J Speech Lang Pathol. 1997;6(3):66-9.

16. Behlau M, Santos LMA, Oliveira G. Crosscultural adaptation and validation of the Voice Handicap Index into Brazilian Portuguese. J Voice. 2011;25(3):354-9.

17. Spearman C. 'Footrule' for measuring correlation. Brit J Psychol. 1906;2:89-108.

18. Spearman C. The proof and measurement of association between two things. Am J Psychol. 1904;15(1):72-101.

19. Gorham-Rowan M, Morris R. Aerodynamic analysis of male-to-female transgender voice. J Voice. 2006;20(2):251-62.

20. Gelfer MP, Schofield KJ. Comparison of acoustic and perceptual measures of voice in male-to-female transsexuals perceived as female versus those perceived as male. J Voice. 2000;14(1):22-33.

21. Davies S. Transgender self-evaluation questionnaire. In: Adler H, Mordaunt M (eds.). Voice and communication therapy for the transgender/ transsexual client: a comprehensive clinical guide. San Diego: Plural Publishing; 2006. p. 485-7.
22. Hancock AB. An ICF perspective on voice-related quality of life of american transgender women. $J$ Voice. 2017;31(1):115.e1-115.e8.

23. T'Sjoen G, Moerman M, Van Borsel J, Feyen E, Rubens $\mathrm{R}$, Monstrey $\mathrm{S}$ et al. Impact of voice in transsexuals. Int J Transgenderism. 2006;9(1):1-7. 